PROCEEDINGS OF THE

AMERICAN MATHEMATICAL SOCIETY

Volume 139, Number 3, March 2011, Pages 1013-1022

S 0002-9939(2010)10515-4

Article electronically published on September 15, 2010

\title{
HIGHER ORDER TURÁN INEQUALITIES FOR THE RIEMANN $\xi$-FUNCTION
}

\author{
DIMITAR K. DIMITROV AND FÁBIO R. LUCAS
}

(Communicated by Walter Van Assche)

ABSTRACT. The simplest necessary conditions for an entire function

$$
\psi(x)=\sum_{k=0}^{\infty} \gamma_{k} \frac{x^{k}}{k !}
$$

to be in the Laguerre-Pólya class are the Turán inequalities $\gamma_{k}^{2}-\gamma_{k+1} \gamma_{k-1} \geq 0$. These are in fact necessary and sufficient conditions for the second degree generalized Jensen polynomials associated with $\psi$ to be hyperbolic. The higher order Turán inequalities $4\left(\gamma_{n}^{2}-\gamma_{n-1} \gamma_{n+1}\right)\left(\gamma_{n+1}^{2}-\gamma_{n} \gamma_{n+2}\right)-\left(\gamma_{n} \gamma_{n+1}-\right.$ $\left.\gamma_{n-1} \gamma_{n+2}\right)^{2} \geq 0$ are also necessary conditions for a function of the above form to belong to the Laguerre-Pólya class. In fact, these two sets of inequalities guarantee that the third degree generalized Jensen polynomials are hyperbolic.

Pólya conjectured in 1927 and Csordas, Norfolk and Varga proved in 1986 that the Turán inequalities hold for the coefficients of the Riemann $\xi$-function. In this short paper, we prove that the higher order Turán inequalities also hold for the $\xi$-function, establishing the hyperbolicity of the associated generalized Jensen polynomials of degree three.

\section{INTRODUCTION}

The real entire function $\psi(x)$ is said to belong to the Laguerre-Pólya class $\mathcal{L P}$ if it can be represented as

$$
\psi(x)=c x^{m} e^{-\alpha x^{2}+\beta x} \prod_{k=1}^{\infty}\left(1+x / x_{k}\right) e^{-x / x_{k}},
$$

where $c, \beta, x_{k}$ are real, $\alpha \geq 0, m$ is a nonnegative integer and $\sum x_{k}^{-2}<\infty$. Similarly, the real entire function $\psi_{1}(x)$ is a function of type I in the Laguerre-Pólya class, written $\psi_{1} \in \mathcal{L P} I$, if $\psi_{1}(x)$ or $\psi_{1}(-x)$ can be represented in the form

$$
\psi_{1}(x)=c x^{m} e^{\sigma x} \prod_{k=1}^{\infty}\left(1+x / x_{k}\right),
$$

Received by the editors January 8, 2010 and, in revised form, March 5, 2010 and March 28, 2010

2010 Mathematics Subject Classification. Primary 33E20, 11M06; Secondary 26D07.

Key words and phrases. Laguerre-Pólya class, Maclaurin coefficients, Turán inequalities, Jensen polynomials, Riemann $\xi$ function.

Research supported by the Brazilian Science Foundations FAPESP under Grants 03/01874-2 and 06/60420-0, CNPq under Grant 305622/2009-9, and CAPES under Grant CAPES/DGU-160.

(C)2010 American Mathematical Society Reverts to public domain 28 years from publication 
where $c$ and $\sigma$ are real, $\sigma \geq 0, m$ a nonnegative integer, $x_{k}>0$, and $\sum 1 / x_{k}<\infty$. The real polynomials with only real zeros are called hyperbolic ones. It is clear that $\mathcal{L P} I \subset \mathcal{L P}$. The functions in $\mathcal{L P}$, and only these, are uniform limits, on compact subsets of $\mathbb{C}$, of hyperbolic polynomials (see, for example, Levin [8, Chapter 8]). Similarly, $\psi \in \mathcal{L P} I$ if and only if it is a uniform limit on the compact sets of the complex plane of hyperbolic polynomials whose zeros are either all positive, or all negative. Thus, the classes $\mathcal{L P}$ and $\mathcal{L P} I$ are closed under differentiation, that is, if $\psi \in \mathcal{L P}$, then $\psi^{(\nu)} \in \mathcal{L P}$ for every $\nu \in \mathbb{N}$ and similarly, if $\psi \in \mathcal{L P} I$, then $\psi^{(\nu)} \in \mathcal{L P} I$. Pólya and Schur [13] proved that if

$$
\psi(x)=\sum_{k=0}^{\infty} \gamma_{k} \frac{x^{k}}{k !}
$$

belongs to $\mathcal{L P}$ and its Maclaurin coefficients $\gamma_{k}=\psi^{k}(0)$ are all nonnegative, then $\psi \in \mathcal{L P} I$. It is worth mentioning that the sequences $\left\{\gamma_{k}\right\}$ of Maclaurin coefficients of $\mathcal{L P} I$-functions are called multiplier sequences, and these are the sequences with the property that, for any $n \in \mathbb{N}$ and every hyperbolic polynomial $\sum_{k=0}^{n} a_{k} x^{k}$, the polynomial $\sum_{k=0}^{n} a_{k} \gamma_{k} x^{k}$ is also a hyperbolic one.

The main reason for the interest in the Laguerre-Pólya class is the fact that it is closely related to the celebrated Riemann hypothesis. Recall that the Riemann $\xi$-function is defined by

$$
\xi(i z)=\frac{1}{2}\left(z^{2}-1 / 4\right) \pi^{-z / 2-1 / 4} \Gamma(z / 2+1 / 4) \zeta(z+1 / 2),
$$

where $\zeta(z)$ is the Riemann $\zeta$-function and $\Gamma(z)$ is the gamma function. It is known that $\xi(z)$ is an entire function of order one. Moreover, it can be represented in the form

$$
\xi(x / 2)=8 \int_{0}^{\infty} \Phi(t) \cos x t d t
$$

where

$$
\Phi(t)=\sum_{n=1}^{\infty}\left(2 n^{4} \pi^{2} e^{9 t}-3 n^{2} \pi e^{5 t}\right) \exp \left(-n^{2} \pi e^{4 t}\right) .
$$

Then

$$
\frac{1}{8} \xi(x / 2)=\sum_{k=0}^{\infty}(-1)^{k} \hat{b}_{k} \frac{x^{2 k}}{(2 k) !} \quad \text { with } \quad \hat{b}_{k}=\int_{0}^{\infty} t^{2 k} \Phi(t) d t, \quad k=0,1, \ldots
$$

On setting $z=-x^{2}$, we obtain the entire function

$$
\xi_{1}(z)=\sum_{k=0}^{\infty} \hat{b}_{k} \frac{z^{k}}{(2 k) !}=\sum_{k=0}^{\infty} \hat{\gamma}_{k} \frac{z^{k}}{k !}, \quad \hat{\gamma}_{k}=\frac{k !}{(2 k) !} \hat{b}_{k}
$$

of order $1 / 2$. Thus, the Riemann hypothesis is equivalent to the statement that the entire function $\xi(z)$ belongs to $\mathcal{L P}$, or equivalently, that $\xi_{1}(z) \in \mathcal{L P} I$. Consequently, any new necessary or sufficient conditions on a function $\psi \in \mathcal{L P}$ are of significant interest.

The simplest necessary condition for a function (2) to be in $\mathcal{L P}$ is that the so-called Turán inequalities

$$
T_{k}:=\gamma_{k}^{2}-\gamma_{k-1} \gamma_{k+1} \geq 0, \quad k \in \mathbb{N},
$$


hold. Proofs of this fact can be found in [13, 10, 11. The most straightforward proof is based on the relation between functions from $\mathcal{L P}$ of the form (21) and their generalized Jensen polynomials, defined by

$$
g_{n, k}(x)=g_{n, k}(\psi ; x):=\sum_{j=0}^{n}\left(\begin{array}{c}
n \\
j
\end{array}\right) \gamma_{k+j} x^{j}, \quad n, k=0,1, \ldots
$$

It is known (see [3, 10]) that $\psi \in \mathcal{L P}$ if and only if the corresponding Jensen polynomials $g_{n, k}(\psi ; x)$ are hyperbolic. Then the fact that the Jensen polynomials $g_{2, k}(x)$ must be hyperbolic immediately yields Turán's inequalities (3). An extension of (3) was obtained in [4] where the inequalities

(5) $H_{k}:=4\left(\gamma_{k}^{2}-\gamma_{k-1} \gamma_{k+1}\right)\left(\gamma_{k+1}^{2}-\gamma_{k} \gamma_{k+2}\right)-\left(\gamma_{k} \gamma_{k+1}-\gamma_{k-1} \gamma_{k+2}\right)^{2} \geq 0, \quad k \in \mathbb{N}$,

were shown to be another necessary condition that the entire function $\psi(x)$, defined by (2), is in $\mathcal{L P}$. We call the new set of inequalities higher order Turán inequalities. The idea of the proof is rather simple and is based on the fact that (5) are necessary conditions in order that the generalized third degree Jensen polynomials $g_{3, k-1}(x)$ are hyperbolic. In fact, it is not difficult to prove that (3) and (5) are also sufficient for $g_{3, k-1}(x)$ to be hyperbolic:

Lemma 1. Let $k \in \mathbb{N}$. Then the real polynomial

$$
g_{3, k-1}(x)=\gamma_{k-1}+3 \gamma_{k} x+3 \gamma_{k+1} x^{2}+\gamma_{k+2} x^{3}
$$

with nonzero leading coefficient $\gamma_{k+2}$ is hyperbolic if and only if the inequalities

$$
\gamma_{k+1}^{2}-\gamma_{k} \gamma_{k+2} \geq 0
$$

and

$$
4\left(\gamma_{k}^{2}-\gamma_{k-1} \gamma_{k+1}\right)\left(\gamma_{k+1}^{2}-\gamma_{k} \gamma_{k+2}\right)-\left(\gamma_{k} \gamma_{k+1}-\gamma_{k-1} \gamma_{k+2}\right)^{2} \geq 0
$$

hold simultaneously.

Let us consider entire functions which are represented by Fourier transforms of even, positive and sufficiently fast decaying kernels $K(t)$,

$$
F(x)=\frac{1}{2} \int_{-\infty}^{\infty} K(t) e^{i x t} d t=\int_{0}^{\infty} K(t) \cos (x t) d t .
$$

Then

$$
F(z)=\sum_{m=0}^{\infty} \frac{(-1)^{m} b_{m}}{(2 m) !} z^{2 m}, \quad \text { with } \quad b_{m}:=\int_{0}^{\infty} t^{2 m} K(t) d t, \quad m=0,1,2, \ldots
$$

Again, the change of variable, $z^{2}=-x$, gives

$$
F_{1}(x):=\sum_{k=0}^{\infty} \gamma_{k} \frac{x^{k}}{k !}=\sum_{k=0}^{\infty} b_{k} \frac{x^{k}}{(2 k) !}, \quad \gamma_{k}:=\frac{k !}{(2 k) !} b_{k} .
$$

Then, obviously, $F \in \mathcal{L P}$ if and only if $F_{1} \in \mathcal{L P} I$. Then the first necessary conditions that these hold are that the corresponding Turán determinants $T_{k}:=$ $\gamma_{k}^{2}-\gamma_{k-1} \gamma_{k+1}$ are nonnegative for all $k \in \mathbb{N}$. Since, obviously,

$$
T_{k}=c_{k}\left|\begin{array}{cc}
b_{k} & (2 k-1) b_{k-1} \\
b_{k+1} & (2 k+1) b_{k}
\end{array}\right|
$$


where $c_{k}:=2 k !(k+1) ! /((2 k) !(2 k+2) !)$, then the Turán inequalities are equivalent to

$$
\widetilde{T_{k}}=(2 k+1) b_{k}^{2}-(2 k-1) b_{k-1} b_{k+1} \geq 0, \quad k \in \mathbb{N} .
$$

Therefore, the simplest necessary conditions for the Riemann hypothesis are that the inequalities

$$
(2 k+1) \hat{b}_{k}^{2}-(2 k-1) \hat{b}_{k-1} \hat{b}_{k+1} \geq 0, \quad k \in \mathbb{N},
$$

hold for the moments of the kernel $\Phi(t)$. In 1927, Pólya 12 conjectured that the inequalities (7) are valid. Pólya's conjecture was proved in 1986 by Csordas, Norfolk and Varga 2]. In addition to the proof, 2 provides some history concerning the previous attempts involving contributions of Hayman [7] and Grosswald [5, 6]. The key idea of the proof in [2] is to first establish the following sufficient condition:

Theorem A. If $K(t)$ is a positive, even, sufficiently smooth and fast decaying kernel, and $\log K(\sqrt{t})$ is concave for $t>0$, that is,

$$
(\log K(\sqrt{t}))^{\prime \prime}<0 \quad \text { for } t>0
$$

then the inequalities (6) hold.

Then Csordas, Norfolk and Varga proved by lengthy, careful and detailed analysis of the kernel $\Phi(t)$ that:

Theorem B. The function $\log \Phi(\sqrt{t})$ is concave for $t>0$.

It is worth mentioning that the inequality (8) is equivalent to

$$
\frac{d}{d t}\left\{\frac{K^{\prime}(t)}{t K(t)}\right\}<0 \quad \text { for } t>0
$$

We refer to 1 for an alternative proof of Theorem A as well as for sufficient conditions on a kernel so that its Fourier transform satisfies the so-called double Turán inequalities.

Preliminaries and history aside, we turn to higher order Turán inequalities for Fourier transforms. First of all, simple calculations show that $H_{k}=d_{k} \widetilde{H}_{k}$, where

$$
d_{k}=\frac{[k !]^{2}[(k+1) !]^{2}}{(2 k) !(2 k+1) ![(2 k+3) !]^{2}}
$$

and

$$
\begin{aligned}
\widetilde{H_{k}}= & 4(2 k+3)\left[(2 k+1) b_{k}^{2}-(2 k-1) b_{k-1} b_{k+1}\right]\left[(2 k+3) b_{k+1}^{2}-(2 k+1) b_{k} b_{k+2}\right] \\
(9) & -(2 k+1)\left[(2 k+3) b_{k} b_{k+1}-(2 k-1) b_{k-1} b_{k+2}\right]^{2} .
\end{aligned}
$$

We consider kernels which possess certain properties:

Definition 1. A function $K: \mathbb{R} \longrightarrow \mathbb{R}$ is called an admissible kernel if it satisfies the following properties:

(i) $K(t)>0$ for $t \in \mathbb{R}$,

(ii) $K(t)$ is analytic in the strip $|\operatorname{Im} z|<\tau$ for some $\tau>0$,

(iii) $K(t)=K(-t)$ for $t \in \mathbb{R}$,

(iv) $K^{\prime}(t)<0$ for $t>0$, and

(v) for some $\varepsilon>0$ and $n=0,1,2, \ldots$,

$$
K^{(n)}(t)=\mathcal{O}\left(\exp \left(-|t|^{2+\varepsilon}\right)\right) \text { as } t \longrightarrow \infty .
$$


It is well known that the kernel $\Phi(t)$ satisfies these properties.

Theorem 1. If $K(t)$ is an admissible kernel with moments $b_{k}=\int_{-\infty}^{\infty} t^{2 k} K(t) d t$ and

$$
(\log K(\sqrt{t}))^{\prime \prime}<0 \quad \text { for } \quad t>0
$$

then $\widetilde{H_{k}} \geq 0$ for every $k \in \mathbb{N}$.

It is quite interesting that the logarithmic concavity of $K(\sqrt{t})$ guarantees not only that the Turán inequalities but also the higher ones hold. Then it follows immediately from Lemma 1] Theorem B and Theorem 1 that

Corollary 1. All third degree Jensen polynomials

$$
g_{3, k-1}(x)=\hat{\gamma}_{k-1}+3 \hat{\gamma}_{k} x+3 \hat{\gamma}_{k+1} x^{2}+\hat{\gamma}_{k+2} x^{3}, \quad k \in \mathbb{N},
$$

associated with the Riemann $\xi$-function, are hyperbolic.

\section{Proof of the main Result}

First we recall some facts concerning the relation between a function in the Laguerre-Pólya class and its Jensen polynomials.

Consider the real entire function $\psi(x)$ defined by (2). Its Jensen polynomials are

$$
g_{n}(x)=g_{n}(\psi ; x):=\sum_{j=0}^{n}\left(\begin{array}{l}
n \\
j
\end{array}\right) \gamma_{j} x^{j}, \quad n=0,1, \ldots
$$

Jensen himself proved that $\psi \in \mathcal{L} \mathcal{P}$ if and only if the corresponding Jensen polynomials $g_{n}(\psi ; x)$ are hyperbolic and that the sequence $\left\{g_{n}(\psi ; x / n)\right\}$ converges locally uniformly to $\psi(x)$. Observe that, for any fixed $k \in \mathbb{N}$, the generalized Jensen polynomials $g_{n, k}(\psi ; x), k=0,1, \ldots$, defined by (4), are the Jensen polynomials associated with $\psi^{k}(x)$, that is,

$$
g_{n, k}(\psi ; x)=g_{n}\left(\psi^{k} ; x\right) .
$$

It is easy to check that the identities

$$
g_{n}^{(\nu)}(x)=(n ! /(n-\nu) !) g_{n-\nu, \nu}(x)
$$

and

$$
g_{n, k}^{(\nu)}(x)=(n ! /(n-\nu) !) g_{n-\nu, k+\nu}(x)
$$

hold. Hence, if $\psi \in \mathcal{L} \mathcal{P}$, then all generalized Jensen polynomials (4) are hyperbolic, and by (10), we conclude that $\mathcal{L P}$ is indeed closed under differentiation.

Lemma 1 is a rather straightforward consequence of a classical result of Hermite. Let

$$
f(x)=x^{n}+a_{n-1} x^{n-1}+\cdots+a_{0}
$$

be a real algebraic polynomial. Let $\alpha_{1}, \alpha_{2}, \ldots, \alpha_{n}$ be its zeros and denote by $S_{0}=n$ and

$$
S_{m}=\alpha_{1}^{m}+\alpha_{2}^{m}+\cdots+\alpha_{n}^{m}, \quad m=1,2,3, \ldots
$$


their Newton sums. Then consider the principal minors of the Hankel matrix of $S_{0}, \ldots, S_{2 n-2}$ :

$$
\Delta_{1}=S_{0}, \Delta_{2}=\left|\begin{array}{ll}
S_{0} & S_{1} \\
S_{1} & S_{2}
\end{array}\right|, \ldots, \Delta_{n}=\left|\begin{array}{ccccc}
S_{0} & S_{1} & S_{2} & \ldots & S_{n-1} \\
S_{1} & S_{2} & S_{3} & \ldots & S_{n} \\
S_{2} & S_{3} & S_{4} & \ldots & S_{n+1} \\
\cdot & \cdot & \cdot & \cdot & \cdot \\
S_{n-1} & S_{n} & S_{n+1} & \ldots & S_{2 n-2}
\end{array}\right| .
$$

Then Hermite's theorem [10] states:

Theorem C. The polynomial $f(x)$ is hyperbolic if and only if the minors $\Delta_{k}, k=$ $1, \ldots, n$ are nonnegative. Moreover, the number of distinct zeros of the polynomial $f(x)$ is equal to the index $k$ of the last nonzero minor $\Delta_{k}$ in the above sequence.

It follows from the proof of Hermite's theorem that if $\Delta_{k+1}=0$, then $\Delta_{k+2}=$ $\cdots=\Delta_{n}=0$.

Proof of Lemma 1. The fact that the inequalities (5) are necessary conditions for the polynomial $g_{3, k-1}(x)$ to be hyperbolic follows from a result of Mařík 9]. Let us apply Hermite's theorem to

$$
g_{3, k-1}(x)=\gamma_{k-1}+3 \gamma_{k} x+3 \gamma_{k+1} x^{2}+\gamma_{k+2} x^{3} .
$$

Since $S_{0}=3$,

$$
S_{1}=-\frac{3 \gamma_{k+1}}{\gamma_{k+2}}, \quad S_{2}=\frac{9 \gamma_{k+1}^{2}}{\gamma_{k+2}^{2}}-\frac{6 \gamma_{k}}{\gamma_{k+2}}, \quad S_{3}=\frac{-27 \gamma_{k+1}^{3}}{\gamma_{k+2}^{3}}+\frac{27 \gamma_{k+1} \gamma_{k}}{\gamma_{k+2}^{2}}-\frac{3 \gamma_{k-1}}{\gamma_{k+2}},
$$

and

$$
S_{4}=\frac{81 \gamma_{k+1}^{4}}{\gamma_{k+2}^{4}}-\frac{108 \gamma_{k+1}^{2} \gamma_{k}}{\gamma_{k+2}^{3}}+\frac{18 \gamma_{k}^{2}}{\gamma_{k+2}^{2}}+\frac{12 \gamma_{k+1} \gamma_{k-1}}{\gamma_{k+2}^{2}}
$$

Then straightforward calculations show that $\Delta_{2}=18\left(\gamma_{k+1}^{2}-\gamma_{k} \gamma_{k+2}\right) / \gamma_{k+2}^{2}$ and $\Delta_{3}=27 H_{k} / \gamma_{k+2}^{4}$, where $H_{k}$ is defined by (5). The statement of Lemma 1 then follows immediately from Hermite's theorem.

Next we recall the following nice formula (cf. [14, Part II, Problem 68]), involving determinants of integrals, which will be the main tool in the proof of Theorem 1 .

Theorem D. Let the functions $f_{1}, f_{2}, g_{1}, g_{2}$ be square integrable in $[0, \infty)$. Then $\left|\begin{array}{l}\int_{0}^{\infty} f_{1}(t) g_{1}(t) d t \int_{0}^{\infty} f_{1}(t) g_{2}(t) d t \\ \int_{0}^{\infty} f_{2}(t) g_{1}(t) d t \int_{0}^{\infty} f_{2}(t) g_{2}(t) d t\end{array}\right|=\frac{1}{2} \int_{0}^{\infty} \int_{0}^{\infty}\left|\begin{array}{l}f_{1}\left(x_{1}\right) f_{1}\left(x_{2}\right) \\ f_{2}\left(x_{1}\right) f_{2}\left(x_{2}\right)\end{array}\right|\left|\begin{array}{l}g_{1}\left(x_{1}\right) g_{1}\left(x_{2}\right) \\ g_{2}\left(x_{1}\right) g_{2}\left(x_{2}\right)\end{array}\right| d x_{1} d x_{2}$.

Proof of Theorem 1, Let $K(t)$ be an admissible kernel. We define

$$
A\left(x_{1}, x_{2}\right)=\left(x_{1}+x_{2}\right) K\left(x_{1}\right) K\left(x_{2}\right)\left\{\left(x_{2}-x_{1}\right)\left|\begin{array}{cc}
\frac{K^{\prime}\left(x_{1}\right)}{x_{1} K\left(x_{1}\right)} & \frac{K^{\prime}\left(x_{2}\right)}{x_{2} K\left(x_{2}\right)} \\
1 & 1
\end{array}\right|\right\},
$$

and, for any pair of nonnegative integers $m$ and $n$,

$$
I_{m, n}=\int_{0}^{\infty} \int_{0}^{\infty} x_{1}^{m} x_{2}^{n} A\left(x_{1}, x_{2}\right) d x_{1} d x_{2} .
$$

The Mean Value Theorem and (8) imply that $A\left(x_{1}, x_{2}\right)>0$ for every $\left(x_{1}, x_{2}\right)$ with $x_{1}, x_{2}>0, x_{1} \neq x_{2}$. Recall that

$$
\widetilde{T_{k}}=(2 k+1) b_{k}^{2}-(2 k-1) b_{k-1} b_{k+1} .
$$


It was proved in [1] that $2 \widetilde{T_{k}}=I_{2 k, 2 k}$. In a similar way, we obtain an explicit expression for the quantity $\widetilde{U_{k}}=(2 k+3) b_{k} b_{k+1}-(2 k-1) b_{k-1} b_{k+2}$. Since

$$
\widetilde{U_{k}}=\left|\begin{array}{cc}
b_{k} & (2 k-1) b_{k-1} \\
b_{k+2} & (2 k+3) b_{k+1}
\end{array}\right|
$$

and integration by parts yields $(2 k-1) b_{k-1}=-\int_{0}^{\infty} t^{2 k-1} K^{\prime}(t) d t$, we have

$$
\widetilde{U_{k}}=\left|\begin{array}{cc}
\int_{0}^{\infty} t^{2 k} K(t) d t & \int_{0}^{\infty} t^{2 k} \frac{-K^{\prime}(t)}{t} d t \\
\int_{0}^{\infty} t^{2 k+4} K(t) d t & \int_{0}^{\infty} t^{2 k+4} \frac{-K^{\prime}(t)}{t} d t
\end{array}\right|
$$

Applying Theorem D with $f_{1}(t)=t^{2 k}, f_{2}(t)=t^{2 k+4}, g_{1}(t)=K(t)$, and $g_{2}(t)=$ $-K^{\prime}(t) / t$, we obtain

$$
\widetilde{U_{k}}=\frac{1}{2}\left[I_{2 k+2,2 k}+I_{2 k, 2 k+2}\right] .
$$

Consider the difference $I_{2 k+2,2 k}-I_{2 k, 2 k+2}$. It follows from the definition of the integrals $I_{m, n}$ that

$$
I_{2 k+2,2 k}-I_{2 k, 2 k+2}=\int_{0}^{\infty} \int_{0}^{\infty} x_{1}^{2 k} x_{2}^{2 k}\left(x_{1}^{2}-x_{2}^{2}\right) A\left(x_{1}, x_{2}\right) d x_{1} d x_{2} .
$$

Denote the last integrand by $G\left(x_{1}, x_{2}\right)$. Since it is obviously antisymmetric with respect to the line $x_{1}=x_{2}$, that is $G\left(x_{1}, x_{2}\right)=-G\left(x_{2}, x_{1}\right)$, then the above integral vanishes and $I_{2 k+2,2 k}=I_{2 k, 2 k+2}$. This yields

$$
\widetilde{U}_{k}=I_{2 k+2,2 k}=I_{2 k, 2 k+2} .
$$

Then, by (9),

$$
\begin{aligned}
\widetilde{H_{k}} & =4(2 k+3) \widetilde{T}_{k} \widetilde{T}_{k+1}-(2 k+1) \widetilde{U}_{k}^{2} \\
& =(2 k+3) I_{2 k, 2 k} I_{2 k+2,2 k+2}-(2 k+1) I_{2 k+2,2 k} I_{2 k, 2 k+2}
\end{aligned}
$$

Consider the difference $\widetilde{J_{k}}:=I_{2 k, 2 k} I_{2 k+2,2 k+2}-I_{2 k+2,2 k} I_{2 k, 2 k+2}$ and represent it in a form of a determinant:

$$
\widetilde{J_{k}}=\left|\begin{array}{cc}
\int_{0}^{\infty} u^{2 k}\left[\int_{0}^{\infty} v^{2 k} A(u, v) d v\right] d u & \int_{0}^{\infty} u^{2 k}\left[\int_{0}^{\infty} v^{2 k+2} A(u, v) d v\right] d u \\
\int_{0}^{\infty} u^{2 k+2}\left[\int_{0}^{\infty} v^{2 k} A(u, v) d v\right] d u & \int_{0}^{\infty} u^{2 k+2}\left[\int_{0}^{\infty} v^{2 k+2} A(u, v) d v\right] d u
\end{array}\right| .
$$

Applying Theorem D with $f_{1}(u)=u^{2 k}, f_{2}(u)=u^{2 k+2}, g_{1}(u)=\int_{0}^{\infty} v^{2 k} A(u, v) d v$, and $g_{2}(u)=\int_{0}^{\infty} v^{2 k+2} A(u, v) d v$ yields

$$
\widetilde{J_{k}}=\frac{1}{2} \int_{0}^{\infty} \int_{0}^{\infty}\left|\begin{array}{cc}
y_{1}^{2 k} & y_{2}^{2 k} \\
y_{1}^{2 k+2} & y_{2}^{2 k+2}
\end{array}\right|\left|\begin{array}{cc}
g_{1}\left(y_{1}\right) & g_{1}\left(y_{2}\right) \\
g_{2}\left(y_{1}\right) & g_{2}\left(y_{2}\right)
\end{array}\right| d y_{1} d y_{2}
$$

which is equivalent to

$$
\widetilde{J_{k}}=\int_{0}^{\infty} \int_{0}^{\infty} y_{1}^{2 k} y_{2}^{2 k} g_{1}\left(y_{1}\right) g_{1}\left(y_{2}\right)\left(y_{1}+y_{2}\right)\left\{\left(y_{2}-y_{1}\right)\left|\begin{array}{cc}
1 & 1 \\
\frac{g_{2}\left(y_{1}\right)}{g_{1}\left(y_{1}\right)} & \frac{g_{2}\left(y_{2}\right)}{g_{1}\left(y_{2}\right)}
\end{array}\right|\right\} d y_{1} d y_{2} .
$$

Since, by the Mean Value Theorem, the expression in the curly brackets is equal to

$$
\left(y_{2}-y_{1}\right)^{2} \Psi^{\prime}(y) \text { with } \Psi(y)=\frac{g_{2}(y)}{g_{1}(y)} \text { and } y \in\left(y_{1}, y_{2}\right),
$$

then $\widetilde{J_{k}} \geq 0$ provided that $\Psi^{\prime}(y) \geq 0$ for every $y \in[0, \infty)$. 
Obviously,

$$
\left(g_{1}(y)\right)^{2} \Psi^{\prime}(y)=\left(g_{1}(y)\right)^{2}\left[\frac{g_{2}(y)}{g_{1}(y)}\right]^{\prime}=g_{2}^{\prime}(y) g_{1}(y)-g_{2}(y) g_{1}^{\prime}(y) .
$$

In what follows we shall denote the partial derivatives $\partial^{i+j} F / \partial x^{i} \partial y^{j}$ of the bivariate function $F(x, y)$ by $\mathcal{D}_{i, j} F(x, y)$ or $F_{i j}(x, y)$.

Since $g_{1}(u)=\int_{0}^{\infty} v^{2 k} A(u, v) d v$ and $g_{2}(u)=\int_{0}^{\infty} v^{2 k+2} A(u, v) d v$, these integrals are uniformly convergent with respect to $y$ in $(0, \infty)$, as are the integrals $\int_{0}^{\infty} v^{2 k} A_{1,0}(y, v) d v$ and $\int_{0}^{\infty} v^{2 k+2} A_{1,0}(y, v) d v$; thus

$$
g_{1}^{\prime}(y)=\int_{0}^{\infty} v^{2 k} A_{1,0}(y, v) d v \quad \text { and } \quad g_{2}^{\prime}(y)=\int_{0}^{\infty} v^{2 k+2} A_{1,0}(y, v) d v .
$$

Hence, the right-hand side of (13) equals

$$
\int_{0}^{\infty} v^{2 k+2} A_{1,0}(y, v) d v \int_{0}^{\infty} v^{2 k} A(y, v) d v-\int_{0}^{\infty} v^{2 k+2} A(y, v) d v \int_{0}^{\infty} v^{2 k} A_{1,0}(y, v) d v
$$

which we write as a determinant:

$$
\left(g_{1}(y)\right)^{2} \Psi^{\prime}(y)=\left|\begin{array}{cc}
\int_{0}^{\infty} v^{2 k} A(y, v) d v & \int_{0}^{\infty} v^{2 k} A_{1,0}(y, v) d v \\
\int_{0}^{\infty} v^{2 k+2} A(y, v) d v & \int_{0}^{\infty} v^{2 k+2} A_{1,0}(y, v) d v
\end{array}\right| .
$$

Now, we apply Theorem D for the last time, with $f_{1}(v)=v^{2 k}, f_{2}(v)=v^{2 k+2}$, $g_{1}(v)=A(y, v)$ and $g_{2}(v)=A_{1,0}(y, v)$, to obtain

$$
2\left(g_{1}(y)\right)^{2} \Psi^{\prime}(y)=\int_{0}^{\infty} \int_{0}^{\infty}\left|\begin{array}{cc}
z_{1}^{2 k} & z_{2}^{2 k} \\
z_{1}^{2 k+2} & z_{2}^{2 k+2}
\end{array}\right|\left|\begin{array}{cc}
A\left(y, z_{1}\right) & A\left(y, z_{2}\right) \\
A_{1,0}\left(y, z_{1}\right) & A_{1,0}\left(y, z_{2}\right)
\end{array}\right| d z_{1} d z_{2},
$$

and the right-hand side is equal to

$$
\int_{0}^{\infty} \int_{0}^{\infty} z_{1}^{2 k} z_{2}^{2 k} A\left(y, z_{1}\right) A\left(y, z_{2}\right)\left(z_{2}+z_{1}\right)\left\{\left(z_{2}-z_{1}\right)\left|\begin{array}{cc}
1 & 1 \\
\frac{A_{1,0}\left(y, z_{1}\right)}{A\left(y, z_{1}\right)} & \frac{A_{1,0}\left(y, z_{2}\right)}{A\left(y, z_{2}\right)}
\end{array}\right|\right\} d z_{1} d z_{2} .
$$

Again the Mean Value Theorem implies that, for any $z_{1}, z_{2}$, there exists $z$ in the interval surrounded by $z_{1}$ and $z_{2}$, such that the expression in the last curly brackets is equal to

$$
\left(z_{2}-z_{1}\right)^{2} \mathcal{D}_{0,1}\left[\frac{A_{1,0}(y, z)}{A(y, z)}\right] .
$$

Summarizing, we see that $\Psi^{\prime}(y)>0$ if

$$
\mathcal{D}_{0,1}\left[\frac{A_{1,0}(y, z)}{A(y, z)}\right]>0 \text { for } y, z \in(0, \infty) .
$$

It remains only to analyze

$$
\mathcal{D}_{0,1}\left[\frac{A_{1,0}(y, z)}{A(y, z)}\right]=\frac{A(y, z) A_{11}(y, z)-A_{1,0}(y, z) A_{0,1}(y, z)}{(A(y, z))^{2}} .
$$


Observe that, if we set

$$
B(y, z):=\left|\begin{array}{cc}
\frac{K^{\prime}(y)}{y} & \frac{K^{\prime}(z)}{z} \\
K(y) & K(z)
\end{array}\right|,
$$

then

$$
\begin{aligned}
& A(y, z)=\left(z^{2}-y^{2}\right) B(y, z), \\
& A_{1,0}=\left(z^{2}-y^{2}\right) B_{1,0}-2 y B, \\
& A_{0,1}=\left(z^{2}-y^{2}\right) B_{0,1}+2 z B,
\end{aligned}
$$

and

$$
A_{1,1}=\left(z^{2}-y^{2}\right) B_{1,1}+2 z B_{1,0}-2 y B_{0,1} .
$$

Substituting these expressions into the numerator on the right-hand side of (14), we obtain

$$
A A_{1,1}-A_{1,0} A_{0,1}=\left(y^{2}-z^{2}\right)^{2}\left(B B_{1,1}-B_{1,0} B_{0,1}\right)+4 y z B^{2} .
$$

The latter function is positive for $y, z \in(0, \infty)$ provided that $L=B B_{1,1}-B_{1,0} B_{0,1}$ is positive. A straightforward calculation immediately yields

$y^{2} z^{2} L=\left[y\left(K^{\prime}(y)\right)^{2}+K(y)\left(K^{\prime}(y)-y K^{\prime \prime}(y)\right)\right]\left[z\left(K^{\prime}(z)\right)^{2}+K(z)\left(K^{\prime}(z)-z K^{\prime \prime}(z)\right)\right]$.

Since

$$
t\left(K^{\prime}(t)\right)^{2}+K(t)\left(K^{\prime}(t)-t K^{\prime \prime}(t)\right)=-(t K(t))^{2}\left[\frac{K^{\prime}(t)}{t K(t)}\right]^{\prime}
$$

then

$$
L=K^{2}(y)\left[\frac{K^{\prime}(y)}{y K(y)}\right]^{\prime} K^{2}(z)\left[\frac{K^{\prime}(z)}{z K(z)}\right]^{\prime} .
$$

On the other hand,

$$
\left[\frac{K^{\prime}(t)}{t K(t)}\right]^{\prime}<0 \text { for all } t>0
$$

which implies $L>0$. Thus,

$$
\widetilde{J}_{k}=I_{2 k, 2 k} I_{2 k+2,2 k+2}-\left[I_{2 k+2,2 k}\right]^{2}>0 .
$$

Then obviously

$$
(2 k+3) I_{2 k, 2 k} I_{2 k+2,2 k+2}-(2 k+1)\left[I_{2 k+2,2 k}\right]^{2}>0,
$$

which is equivalent to $\widetilde{H_{k}}>0$. This completes the proof of the theorem.

It is worth noting that we have proven that the logarithmic concavity of the kernel $K(\sqrt{t})$ on the positive real axes implies the stronger inequalities $\widetilde{H}_{k}>8 \widetilde{T}_{k} \widetilde{T}_{k+1}$.

It is also of interest to point out that there are kernels which satisfy the requirement $(\log K(\sqrt{t}))^{\prime \prime}<0, t>0$, whose cosine transforms $\int_{0}^{\infty} K(t) \cos z t d t$ do not belong to the Laguerre-Pólya class. A simple example is given by $K(t)=$ $\exp \left(-t^{3}\right)$.

\section{ACKNOWLEDGEMENT}

We thank the referee, whose valuable remarks helped us to improve the presentation of the paper. 


\section{REFERENCES}

[1] G. Csordas and D. K. Dimitrov, Conjectures and theorems in the theory of entire functions, Numer. Algorithms 25 (2000), 109-122. MR1827149(2002a:30042)

[2] G. Csordas, T. S. Norfolk and R. S. Varga, The Riemann Hypothesis and the Turán inequalities, Trans. Amer. Math. Soc. 296 (1986), 521-541. MR846596 (87i:11109)

[3] G. Csordas and R. Varga, Necessary and sufficient conditions and the Riemann hypothesis, Adv. Appl. Math. 11 (1990), 328-357. MR1061423 (91d:11107)

[4] D. K. Dimitrov, Higher order Turán inequalities, Proc. Amer. Math. Soc. 126 (1998), 20332037. MR1459117 (99g:33021)

[5] E. Grosswald, Generalization of a formula of Hayman, and its application to the study of Riemann's zeta function, Illinois J. Math. 10 (1966), 9-23. MR0186797 (32:4252)

[6] E. Grosswald, Correction and completion of the paper "Generalization of a formula of Hayman", Illinois J. Math. 13 (1969), 276-280. MR0240290 (39:1639)

[7] W. Hayman, A generalization of Stirling's formula, J. Reine Angew. Math. 196 (1956), 67-95. MR0080749 (18:293f)

[8] B. Ya. Levin, Distribution of zeros of entire functions, Transl. Math. Mono. vol. 5, Amer. Math. Soc., Providence, RI, 1964; revised ed. 1980. MR.589888 (81k:30011)

[9] J. Mařík, On polynomials, all of whose zeros are real, Casopis. Pěst. Mat. 89 (1964), 5-9. MR0180548 (31:4782)

[10] N. Obrechkoff, Zeros of Polynomials, Publ. Bulg. Acad. Sci., Sofia, 1963 (in Bulgarian); English translation (by I. Dimovski and P. Rusev) published by The Marin Drinov Academic Publishing House, Sofia, 2003.

[11] N. Obrechkoff, Verteilung und Berechnung der Nullstellen reeller Polynome, VEB Deutscher Verlag der Wissenschaften, Berlin, 1963. MR.0164003 (29:1302)

[12] G. Pólya, Über die algebraisch-funcktionentheoretischen Untersuchungen von J. L. W. V. Jensen, Kgl. Danske Vid. Sel. Math.-Fys Med 7 (1927), 3-33.

[13] G. Pólya and J. Schur, Über zwei Arten von Faktorenfolgen in der Theorie der algebraischen Gleichungen, J. Reine Angew. Math. 144 (1914), 89-113.

[14] G. Pólya and G. Szegő, Aufgaben und Lehrsätze aus der Analysis, Springer-Verlag, Berlin, 1925; English translation: Problems and Theorems in Analysis, Vol. II, Springer-Verlag, Berlin, 1976. MR0396134 (53:2)

Departamento de Ciências de Computação e Estatística, ibilce, Universidade Estadual Paulista, 15054-000 São José do Rio Preto, SP, Brazil

E-mail address: dimitrov@ibilce.unesp.br

Departamento de matemática Aplicada, imeCC, UniCAMP, 13083-859 Campinas, SP, BRAZIL

E-mail address: fabio25jk@hotmail.com 